\title{
Analysis of Radiographic Spinopelvic Parameters in Patients With Degenerative Lumbar Kyphoscoliosis
}

\section{Ge Rile}

Peking University Third Hospital https://orcid.org/0000-0001-9759-8843

\section{Peng Yang}

Peking University International Hospital

Xin Liu

Peking University International Hospital

\section{Bingtao Wen}

Peking University International Hospital

\section{Zhaoqing Guo}

Peking University Third Hospital

\section{zhongqiang Chen ( $\nabla$ czqpkuth@sina.com )}

Department of Orthopedics, PeKing University Third Hospital. 49 North Garden Road, Haidian District, Beijing, 100191, China. https://orcid.org/0000-0003-3160-5283

\section{Research article}

Keywords: degenerative lumbar kyphoscoliosis, sagittal balance, LL-TK, PI-LL

Posted Date: September 22nd, 2020

DOI: https://doi.org/10.21203/rs.3.rs-74784/v1

License: (c) (i) This work is licensed under a Creative Commons Attribution 4.0 International License.

Read Full License 


\section{Abstract}

Purpose: To analyze the relationships between coronal and sagittal spinopelvic parameters in degenerative lumbar kyphoscoliosis (DLKS).

Methods: We enrolled 75 patients with DLKS for a radiographic study between January 2016 and September 2018. Correlations between coronal and sagittal spinopelvic radiographic parameters were analyzed. Then patients were divided into two groups: sagittal balanced group ( $S V A<=5 \mathrm{~cm}, 30$ patients) and sagittal imbalanced group (SVA> 5cm, 45 patients), and relevant parameters were compared.

Results: The Cobb angle and lumbar lordosis of the DLKS patients were $24.87 \pm 11.59^{\circ}$ and $17.26 \pm$ $12.24^{\circ}$, respectively. The average age was 68 years old (range: $42-82$ ), and the sex ratio was 2.6:1 (female:54 patients; male: 21 patients). 50 patients (66.7\%) located convexity of the curve at left side, while 25 patients (33.3\%) at right side. The Cobb angle correlated with LL-TK $(r=-0.228, p=0.049)$, LL ( $r$ $=-0.255, p=0.027)$ and SS $(r=-0.232, p=0.045)$. There were significant differences in PI-LL $(t=-3.484, P$ $=0.001), \operatorname{LL}-T K(t=2.354, P=0.023), P I(t=-3201, P=0.002)$ and $P T(t=-2.521, P=0.014)$ between sagittal balanced and imbalanced group.

Conclusions: In degenerative lumbar kyphoscoliosis, there are some correlations between coronal and sagittal spinopelvic parameters. Moreover, PI-LL, LL-TK, PI, PT were significantly different between sagittal balanced and imbalanced DLKS patients.

\section{Background}

Degenerative lumbar kyphoscoliosis (DLKS) is a complex three-dimensional (3D) deformity of the spine, involving deviations in the coronal plane, modifications of the sagittal profile, and rotations in the transverse plane. In addition to coronal deformity and vertebral rotation, the aging process also affects the sagittal profile, leading to lumbar degenerative kyphosis and sagittal imbalance, namely degenerative lumbar kyphoscoliosis ${ }^{[1-3]}$. Adult scoliosis is defined as spinal deformity with a scoliotic angle of over 10 degrees in skeletally-mature patients, while degenerative lumbar kyphosis (DLK) is thought to be a sagittal imbalance due to lumbar kyphosis or marked loss of lumbar lordosis.

Management of patients with rigid DLKS remains one of the most challenging issues in the field of spinal surgery, due to the complexity and the diversity of structural pathologies and clinical presentations, especially in our aging society. Patients with DLKS often present with 3D rigid curvatures that can be accelerated by disc degeneration, osteophyte formation and osteoporotic vertebrae.

During the past few decades, spinal surgeons have focused on treatment options for DLKS. Surgical treatment has been reported to be the preferred option compared with conservative treatment ${ }^{[4]}$, the goals of which are to correct three-dimensional deformities, achieve solid fusion, relieve pain, and prevent deformity progression. However, to figure out pathogenesis and to formulate surgical strategies, the 
relationships among coronal, sagittal and axial planes should be studied in DLKS patients. To the best of our knowledge, there were few studies on this subject.

The purpose of this study is to analyze the relationships between coronal and sagittal spinopelvic parameters in degenerative lumbar kyphoscoliosis.

\section{Materials And Methods}

The study was conducted in accordance with the Declaration of Helsinki. Informed written consent was obtained from all patients prior to enrollment.

We enrolled 75 patients with degenerative lumbar deformities between January 2016 to September 2018 . Complete radiographic evaluations were performed using a full-length 36 -inch standing lateral radiograph, with the arms held at $60^{\circ}$ of forward flexion and the hips and knees fully extended.

Inclusion criteria were as follows: presentation with sagittal imbalance due to lumbar kyphosis or marked loss of lumbar lordosis; scoliotic angle of over 10 degrees in DLKS patients. Exclusion criteria were as follows: history of trauma or surgery to the spine, pelvis or other locations; any co-morbidity that may affect the spinopelvic alignment, such as pelvic deformities, leg length discrepancy and spondylolisthesis; and incompleteness of patient's information or absence of some measurements.

Coronal Cobb angle is defined as the angle between the upper endplate of the superior end vertebra and the lower endplate of the inferior end vertebra. L3 tilt is defined as the angle between the upper endplate of $L 3$ and the horizontal line. Coronal balance distance (CBD) defined as the horizontal distance between the midpoint of $\mathrm{C} 7$ and the center of the pelvis on coronal plane.

Sagittal balance was determined by measuring the sagittal vertical axis (SVA) with a plumb line from the center of the $\mathrm{C} 7$ vertebral body to the posterior sacral prominence on the lateral radiograph. A regional sagittal modifier was included to describe each of the three regions of the spine: thoracic kyphosis (TK), thoracolumbar junctional angle (TLJA) and lumbar lordosis (LL). The main TK was measured from the T4 superior end plate to T12 inferior end plate. The TLJA was measured from the T11 superior end plate to $L 1$ inferior end plate. The LL was measured from the L1 superior end plate to $S 1$ superior end plate by the Cobb method. In terms of TK, TLJA and LL, we defined lordosis as positive and kyphosis as negative.

As for the pelvic parameters, pelvic incidence, sacral slope and pelvic tilt were measured in each whole spine lateral view. Pelvic incidence (PI) was defined as the angle between the line perpendicular to the sacral plate, and the line connecting the midpoint of the sacral plate to the bicoxofemoral axis. The sacral slope (SS) is defined as the angle between the S1 superior end plate and the horizontal. Pelvic tilt (PT) was defined as the angle between a vertical line originating at the center of the bicoxofemoral axis and a line drawn between the same point and the middle of the superior end plate of S1. Apical vertebral rotation was accessed by Nash-Moe grading scale ${ }^{[5]}$. 
Statistical analysis was performed using SPSS $20.0 \mathrm{~K}$ (SPSS Inc., Chicago, IL, USA). A probability value of less than 0.05 was considered significant. The correlations between spinopelvic parameters were determined using the Pearson correlation coefficient. The independent two-sample t test was used to compare variables between groups.

\section{Results}

For DLKS patients, typical degenerations were often observed in both coronal and sagittal planes (Fig. 1). The average age of DLKS patients was 68 years old (range: $42-82$ years old), and the sex ratio was 2.6:1 (female:54 patients; male: 21 patients). 50 patients (66.7\%) located convexity of the curve at left side, while 25 patients $(33.3 \%)$ at right side. The median Cobb $\left(^{\circ}\right)$ was $24.87 \pm 11.59$, the median $\operatorname{LL}\left({ }^{\circ}\right)$ was $17.26 \pm 12.16$, the median segments number of curve was 4.53 (range: $3-7$ ) and the median apical vertebra rotation was $3^{\circ}$ (range: $\left.1-5\right)$ (Table 1 ). As shown in Fig. 2, most of apical disc/vertebrae located at L1/2, L2, L2/3 and L3 (84\%).

Table 1

General characteristics and radiographic parameters in DLKS patients.

\begin{tabular}{|llll|}
\hline & Median & Minimum & Maximum \\
\hline Age (years old) & 68 & 42 & 82 \\
\hline segments & 4.53 & 3 & 7 \\
\hline Cobb $\left(^{\circ}\right)$ & 24.87 & 10.4 & 61.00 \\
\hline CBD $(\mathrm{mm})$ & 17.50 & 0.00 & 66.71 \\
\hline L3 tilt $\left(^{\circ}\right)$ & 11.09 & 0.40 & 29.00 \\
\hline Apical rotation & 3 & 1 & 5 \\
\hline C7-SVA (mm) & 64.60 & 0.00 & 174.70 \\
\hline $\mathrm{PI}-\mathrm{LL}\left(^{\circ}\right)$ & 26.27 & 0.90 & 67.40 \\
\hline $\mathrm{LL}-\mathrm{TK}\left({ }^{\circ}\right)$ & -3.23 & -29.10 & 30.50 \\
\hline $\mathrm{LL}\left({ }^{\circ}\right)$ & 17.26 & -14.00 & 29.50 \\
\hline $\mathrm{TK}\left({ }^{\circ}\right)$ & -20.49 & -54.80 & 0.60 \\
\hline $\mathrm{TLJA}\left({ }^{\circ}\right)$ & -10.75 & -34.40 & 8.20 \\
\hline $\mathrm{PI}\left({ }^{\circ}\right)$ & 43.53 & 22.00 & 75.00 \\
\hline $\mathrm{PT}\left({ }^{\circ}\right)$ & 23.12 & 6.00 & 60.00 \\
\hline $\mathrm{SS}\left(^{\circ}\right)$ & 20.20 & -8.00 & 38.00 \\
\hline
\end{tabular}


We studied correlation coefficients between coronal and sagittal parameters in DLKS patients (Table 2). The Cobb angle correlated with LL-TK $(r=-0.228, p=0.049)$, LL $(r=-0.255, p=0.027)$ and SS $(r=-0.232$, $p=0.045)$ (Fig. 3). We hypothesize that for patients with degenerative lumbar kyphoscoliosis, interrelated degenerations result in some correlations between coronal and sagittal spinopelvic parameters.

Table 2

Correlation coefficients between coronal and sagittal parameters in DLKS patients.

\begin{tabular}{|c|c|c|c|c|c|c|c|c|c|c|}
\hline DLKS & & C7-SVA & PI-LL & LL-TK & LL & TK & TLJA & PI & PT & SS \\
\hline \multirow[t]{2}{*}{ Cobb } & $r$ & 0.128 & 0.223 & -0.228 & -0.255 & 0.018 & -0.199 & 0.022 & 0.197 & -0.232 \\
\hline & $\mathrm{p}$ & 0.274 & 0.054 & $0.049 *$ & 0.027 * & 0.875 & 0.087 & 0.850 & 0.090 & $0.045^{*}$ \\
\hline \multirow[t]{2}{*}{ CBD } & $r$ & 0.184 & 0.099 & 0.077 & -0.077 & 0.152 & 0.213 & 0.053 & 0.048 & 0.015 \\
\hline & $\mathrm{p}$ & 0.114 & 0.396 & 0.510 & 0.510 & 0.194 & 0.067 & 0.654 & 0.684 & 0.895 \\
\hline \multirow[t]{2}{*}{ L3 tilt } & $r$ & -0.020 & 0.107 & -0.082 & -0.135 & 0.049 & -0.166 & -0.004 & 0.123 & -0.163 \\
\hline & $\mathrm{p}$ & 0.864 & 0.360 & 0.483 & 0.247 & 0.679 & 0.154 & 0.972 & 0.294 & 0.163 \\
\hline \multicolumn{11}{|c|}{$\begin{array}{l}\text { DLKS, degenerative lumbar kyphoscoliosis; LL, lumbar lordosis; TK, thoracic kyphosis; SS, sacral } \\
\text { slope; TLJA, thoracolumbar junctional angle; PI, pelvic incidence; PT, pelvic tilt; C7-SVA, C7 sagittal } \\
\text { vertical axis; CBD, coronal balance distance. }\end{array}$} \\
\hline
\end{tabular}

Patients with degenerative lumbar kyphosis were divided into two groups: sagittal balanced group (SVA< $=5 \mathrm{~cm}, 30$ patients) and sagittal imbalanced group (SVA $>5 \mathrm{~cm}, 45$ patients). Coronal and sagittal spinopelvic radiographic parameters were compared between two groups. There are significant differences in PI-LL ( $t=-3.484, P=0.001)$,

LL-TK $(\mathrm{t}=2.354, \mathrm{P}=0.023), \mathrm{PI}(\mathrm{t}=-3201, \mathrm{P}=0.002)$ and $\mathrm{PT}(\mathrm{t}=-2.521, \mathrm{P}=0.014) .($ Table 3$)$ 
Table 3

Comparison of all parameters between sagittal balanced group and sagittal imbalanced group.

\begin{tabular}{|lllll|}
\hline & sagittal balanced group & sagittal imbalanced group & t & p \\
\hline age & $66.93 \pm 6.51$ & $69.76 \pm 7.74$ & -1.646 & 0.104 \\
\hline Cobb & $25.11 \pm 11.51$ & $24.71 \pm 11.90$ & 0.145 & 0.885 \\
\hline CBD & $14.13 \pm 11.67$ & $19.75 \pm 16.01$ & -1.651 & 0.103 \\
\hline L3 tilt & $11.85 \pm 6.69$ & $10.59 \pm 7.25$ & 0.764 & 0.447 \\
\hline PI-LL & $19.76 \pm 11.17$ & $30.62 \pm 15.81$ & -3.484 & $0.001^{*}$ \\
\hline LL-TK & $1.11 \pm 14.52$ & $-6.12 \pm 10.44$ & 2.354 & $0.023^{*}$ \\
\hline LL & $19.31 \pm 12.86$ & $15.89 \pm 11.75$ & 1.187 & 0.239 \\
\hline TK & $-18.20 \pm 11.46$ & $-22.02 \pm 13.34$ & 1.284 & 0.203 \\
\hline TLJA & $-12.12 \pm 8.25$ & $-9.83 \pm 8.73$ & -1.139 & 0.259 \\
\hline PI & $39.07 \pm 7.88$ & $46.51 \pm 10.98$ & -3.201 & $0.002^{*}$ \\
\hline PT & $19.87 \pm 7.01$ & $25.29 \pm 10.28$ & -2.521 & $0.014^{*}$ \\
\hline SS & $18.67 \pm 10.21$ & $21.22 \pm 7.46$ & -1.252 & 0.214 \\
\hline LL, lumbar lordosis; TK, thoracic kyphosis; SS, sacral slope; TLJA, thoracolumbar junctional angle; PI, \\
\hline pelvic incidence; PT, pelvic tilt; C7-SVA, C7 sagittal vertical axis; CBD, coronal balance distance.
\end{tabular}

\section{Discussion}

In this study, coronal parameters showed some correlations with sagittal spinopelvic parameters in the DLKS group, suggesting a similar etiology between coronal and sagittal deformities. Characteristic lumbar degenerative pathology includes spinal stenosis, segmental instability (olisthesis and rotatory subluxation), and malalignment of the spine in the sagittal, coronal, and axial planes ${ }^{[6-9]}$. Patients with degenerative lumbar scoliosis (DLS) share a common presentation, with degenerative changes of the spinal motion segment affecting the intervertebral disc and facet joints. DLS has been reported to be triggered by asymmetrical disc degeneration ${ }^{[10,11]}$. Jimbo et al ${ }^{[12]}$ suggested that disc degeneration should be evaluated as a predictor of curvature progression in DLS. Asymmetric loading coupled with degeneration potentiates a vicious cycle of enhanced curve progression ${ }^{[13]}$. Compared with DLS, degenerative lumbar kyphosis has a higher incidence and is associated with more serious clinical outcomes. Jang et al. ${ }^{[14]}$ found that spinal degeneration in DLK patients included disc narrowing, spondylolisthesis, hyperosteogeny, osteophyte formation, hypertrophy of facet joints, osteoporosis, and atrophy of lumbar extensor muscles. They further speculated that serious degeneration of spinal 
structures, such as intervertebral discs, vertebrae or facet joints, was a major cause of DLK. One study demonstrated that the lower end vertebral disc degeneration strongly correlates with sagittal imbalance in patients with DLS, suggesting that disc degeneration may be a risk factor for sagittal imbalance. Disc degeneration strongly correlated with sagittal malalignment, as demonstrated by a more positive SVA, decreased TK and LL, suggesting explanations for low quality of life in elderly patients with DLS [13]. In brief, degenerations originating from intervertebral disc and facet joints induce spinal coronal and sagittal imbalance.

Degenerative lumbar kyphoscoliosis, including coronal, sagittal and axial deformities, was extremely serious in adult lumbar deformities. In fact, some studies focusing on adolescent idiopathic scoliosis revealed that most coronal and sagittal parameters were not significantly correlated, and coronal deformity types did not change the global sagittal postural patterns ${ }^{[15]}$. In our study, we were impressed by the mismatching between coronal scoliosis and sagittal morphological parameters. One reason for this result may be the differences of pathogenesis. Degenerative lumbar scoliosis mainly involves degenerative disc disease, facet incompetence, and hypertrophy of the ligamenta flava, while degenerative lumbar kyphosis results from atrophy and fatty changes of the lumbar extensor muscles and wedging changes of vertebrae in spite of degenerative disc disease. Particular life-styles, such as the prolonged crouched posture during agricultural work and certain activities of daily living play important roles in the process of DLK development. Another reason may be that patients enrolled in our study all had local or global sagittal imbalance because of degenerative lumbar kyphosis. To maintain sagittal balance, co-adjustment of each sagittal spinopelvic parameters lead to close interrelations, covering the effects of scoliosis. The third reason is that complex deformities might give rise to these apparent inconsistencies: severe coronal deformities without corresponding sagittal deformities. With the aging process, we begin to see deformities in coronal and sagittal, such as subluxation, spondylolisthesis, hyperlordosis, and hypolordosis. Gradually, vertebral rotation, thoracic and pelvic compensation alter sagittal balance, inducing relatively moderate changes of sagittal morphology.

As noted in the study of Glassman et al. ${ }^{[16]}$, quality of life in patients with adult spinal deformity was substantially related to sagittal deformity, not to coronal deformity. Between sagittal balanced and imbalanced DLKS patients, we found that PI-LL, LL-TK, PI, PT were significantly different. As we know, Schwab et al. ${ }^{[17]}$ brought out standardized evaluation of the sagittal plane and the goal for correction surgery in adult spinal deformity: SVA $<50 \mathrm{~mm}, \mathrm{PI}-\mathrm{LL}<10^{\circ}$ and $\mathrm{PT}<25^{\circ}$, which had been widely used in clinical practice. Moreover, another study ${ }^{[18]}$ demonstrated that LL-TK could be a good predictor for sagittal balance in Chinese elderly people, which reflected the regional compensatory mechanism and significantly associated with SVA.

Our study was limited by small sample size: if more patients were included, our findings may be validated. In our future study, clinical outcomes, like Japanese Orthopedics Association scale (JOA), Visual Analog Score (VAS) and Oswestry Disability Index (ODI) should be included for the analysis between functional scores and radiological parameters. Nevertheless, we wish to consider this study as 
the first series of explorations focusing on the relationships between degenerative lumbar coronal, sagittal, and axial deformities, in order to properly make treatment plans for degenerative lumbar kyphoscoliosis.

\section{Conclusion}

In degenerative lumbar kyphoscoliosis, there are some correlations between coronal and sagittal spinopelvic parameters. Weak or moderate but significant correlations between Cobb angle and LL-TK, LL or SS. Moreover, PI-LL, LL-TK, PI, PT were significantly different between sagittal balanced and imbalanced DLKS patients.

\section{Abbreviations}

DLKS: degenerative lumbar kyphoscoliosis; DLK: degenerative lumbar kyphosis; 3D: three-dimensional; CBD: coronal balance distance; SVA: sagittal vertical axis; TK: thoracic kyphosis; TLJA: thoracolumbar junctional angle; LL: lumbar lordosis; PI: pelvic incidence; PT: pelvic tilt; SS: sacral slope.

\section{Declarations}

\section{Conflicts of interest}

There are no conflicts of interest.

\section{Acknowledgements}

None

\section{Funding}

No funding was received.

\section{Availability of data and materials}

The datasets generated and analyzed during the current study are available from the corresponding author on reasonable request.

\section{Authors' contributions}

All authors contributed to the conception and design of the study, acquisition and analysis of data, and manuscript drafting and revision. All authors read and approved the final manuscript.

\section{Ethics approval and consent to participate}


All procedures performed in studies involving human participants were in accordance with the ethical standards of the Institutional Review Board of Ethics Committee of Peking University Third Hospital and with the 1964 Helsinki declaration and its later amendments or comparable ethical standards. The informed consent was orally obtained from patients, but not by a form. Additionally, all the patients' data were anonymously tagged for analyses.

\section{Consent for publication}

Not applicable

\section{Competing interests}

The authors declare that they have no competing interests.

\section{References}

1. Toyone T, Shiboi R, Ozawa T, et al. Asymmetrical pedicle subtraction osteotomy for rigid degenerative lumbar kyphoscoliosis. Spine. 2012;37:1847-52.

2. Lafage V, Schwab F, Patel A, et al. Pelvic tilt and truncal inclination: two key radiographic parameters in the setting of adults with spinal deformity. Spine. 2009;34:E599-606.

3. Bao H, Zhu F, Liu Z, et al. Coronal curvature and spinal imbalance in degenerative lumbar scoliosis: disc degeneration is associated. Spine. 2014;39:E1441-7.

4. Smith JS, Klineberg E, Schwab F, et al. Change in classification grade by the SRS-Schwab adult spinal deformity classification predicts impact on health-related quality of life measures: prospective analysis of operative and nonoperative treatment. Spine. 2013;38:1663-71.

5. Nash CL Jr, Moe JH. A study of vertebral rotation. J Bone Joint Surg Am. 1969;51(2):223-9.

6. Ploumis A, Transfledt EE, Denis F. Degenerative lumbar scoliosis associated with spinal stenosis. Spine J. 2007;7:428-36.

7. Vanderpool DW, James JI, Wynne-Davies R. Scoliosis in the elderly. J Bone Joint Surg Am. 1969;51:446-55.

8. Hong JY, Suh SW, Modi HN, et al. The prevalence and radiological findings in 1347 elderly patients with scoliosis. J Bone Joint Surg Br. 2010;92:980-3.

9. Kebaish KM, Neubauer PR, Voros GD, et al. Scoliosis in adults aged forty years and older: prevalence and relationship to age, race, and gender. Spine. 2011;36:731-6.

10. Kobayashi T, Atsuta Y, Takemitsu M, et al. A prospective study of de novo scoliosis in a community based cohort. Spine. 2006;31:178-82.

11. Murata $\mathrm{Y}$, Takahashi $\mathrm{K}$, Hanaoka $\mathrm{E}$, et al. Changes in scoliotic curvature and lordotic angle during the early phase of degenerative lumbar scoliosis. Spine. 2002;27:2268 - 2273.

12. Jimbo S, Kobayashi T, Aono K, et al. Epidemiology of degenerative lumbar scoliosis: a community based cohort study. Spine. 2012;37:1763-70. 
13. Kotwal S, Pumberger M, Hughes A, et al. Degenerative scoliosis: a review. HSS J. 2011;7:257-64.

14. Jang J, Lee SH, Min JH, et a1 (2007) Lumbar degenerative kyphosis: radiologic analysis and classifications. Spine 32:2694-2699.

15. Panpan $\mathrm{H}, \mathrm{Miao} Y$, Xiaoguang $\mathrm{L}$, et al. Analysis of the relationship between coronal and sagittal deformities in adolescent idiopathic scoliosis. Eur Spine J. 2016;25:409-16.

16. Glassman SD, Bridwell K, Dimar JR, Horton W, Berven S, Schwab F. (2005) The impact of positive sagittal balance in adult spinal deformity. Spine 30:2024-9. doi: 10.1097/01. brs.0000179086.30449.96.

17. Schwab F, Ungar B, Lafage V, et al. Scoliosis Research Society-Schwab Adult Spinal Deformity Classification. Spine. 2012;37(12):1077-82.

18. Yang $C$, Yang $M$, Wei $X$, et al. Lumbar lordosis minus thoracic kyphosis: a novel regional predictor for sagittal balance in elderly populations. Spine. 2016;41(5):399-403.

\section{Figures}




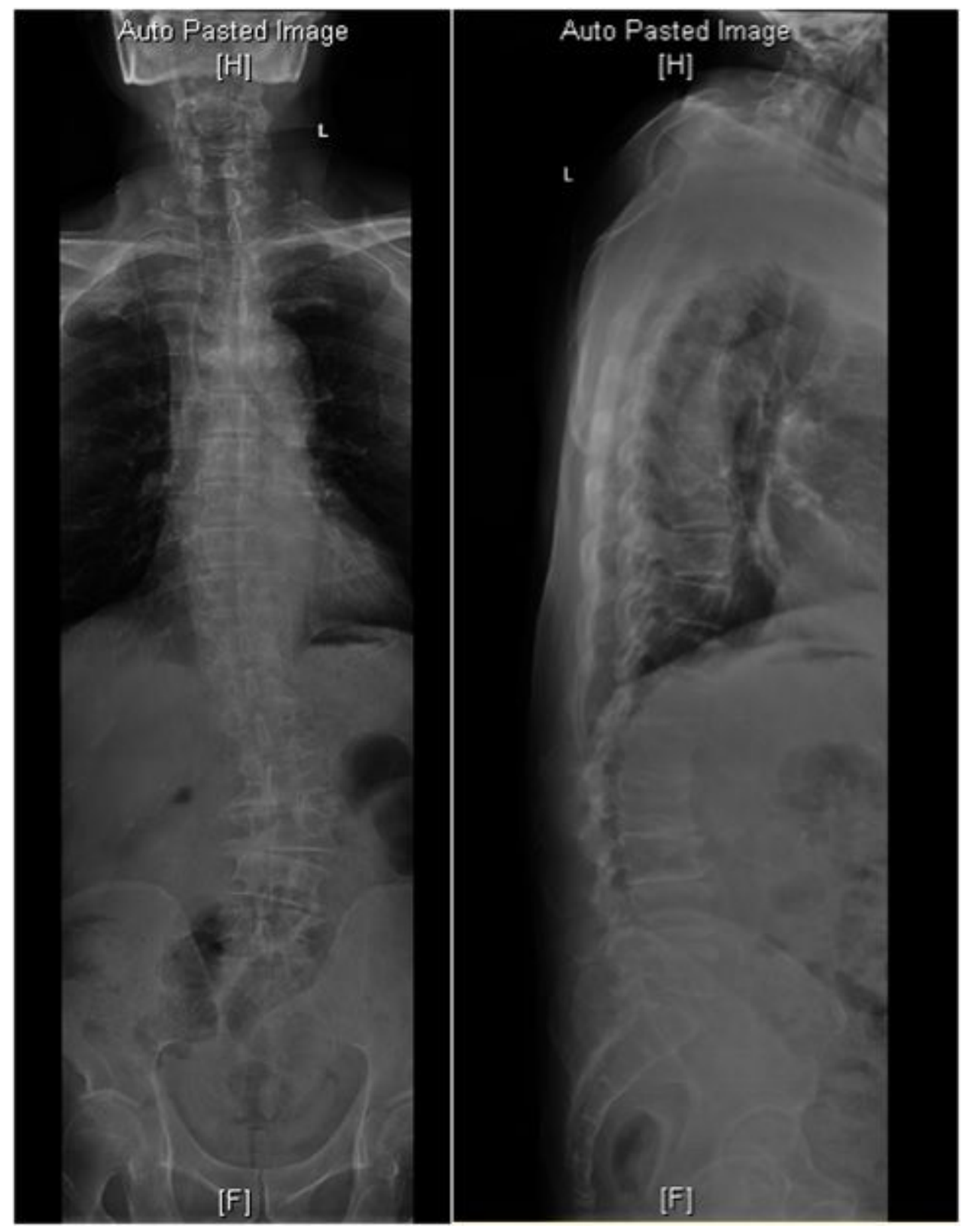

\section{Figure 1}

Typical degenerations in both coronal and sagittal planes: asymmetrical disc narrowing, subluxation, osteophyte formation, hypertrophy of facet joints, spondylolisthesis and osteoporosis. Coronal and sagittal degeneration in the spine both originate from intervertebral discs and bilateral facet joints. 


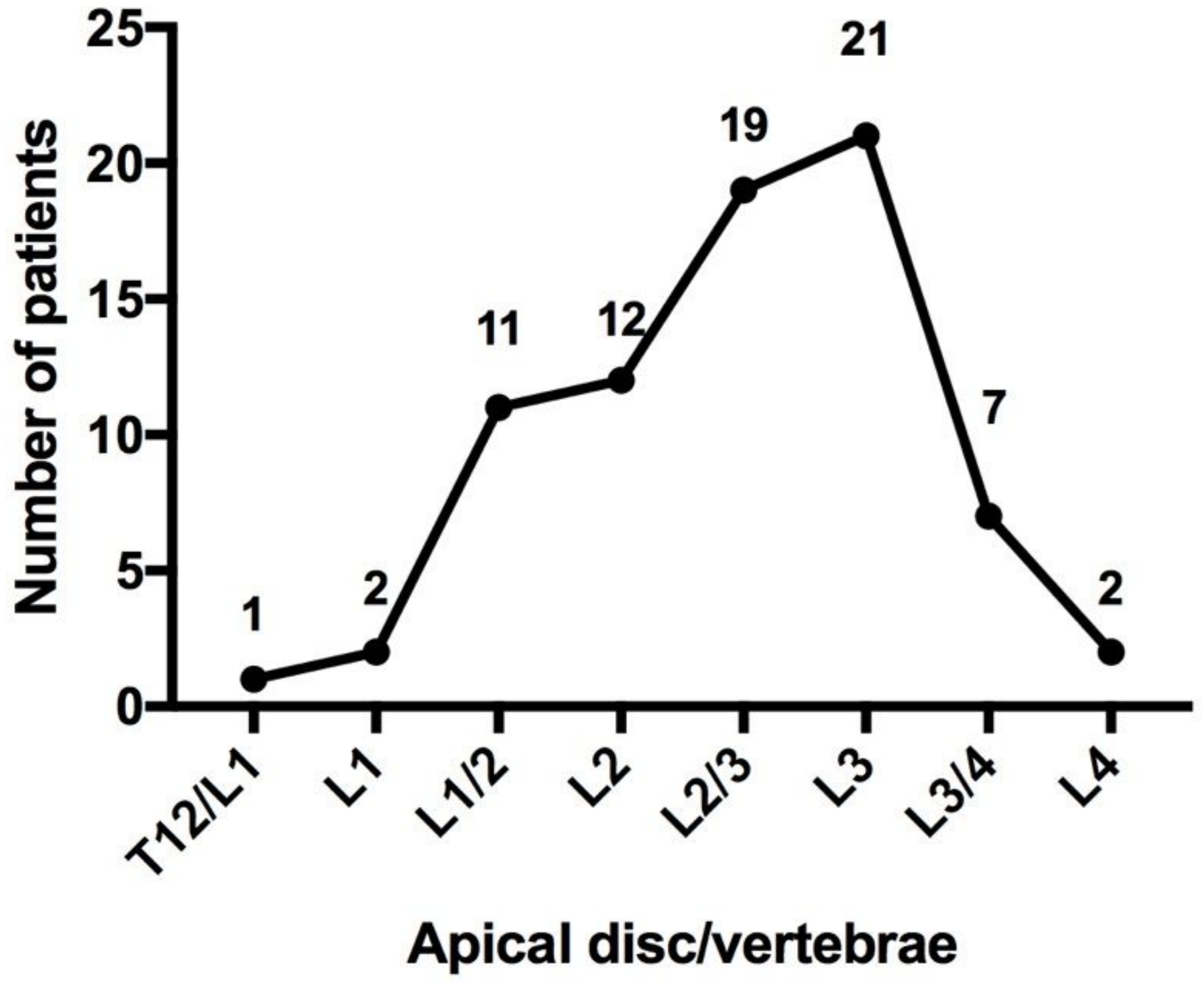

Figure 2

Location of apical disc or vertebrae in DLKS patients.
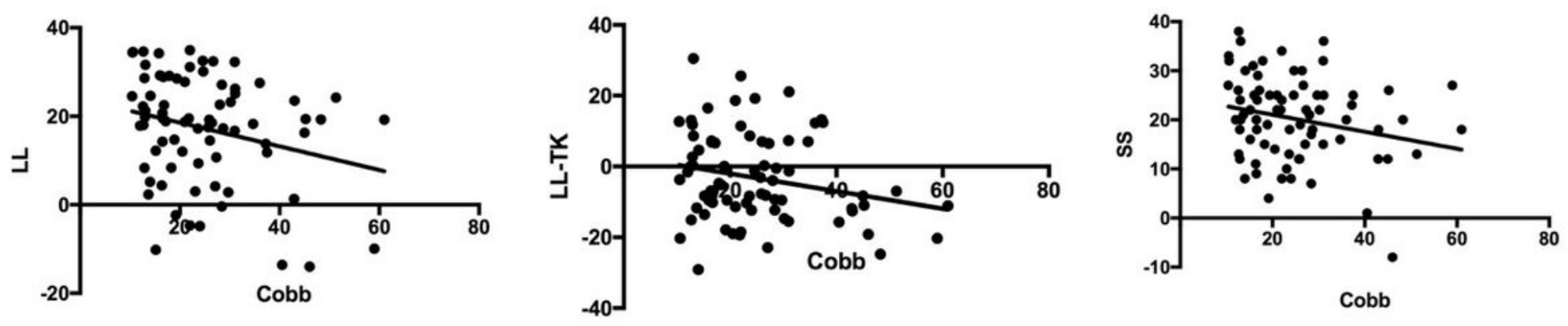

Figure 3

The coronal Cobb angle correlated with sagittal LL, LL-TK and SS. 